\title{
Design and Verification of a Blood Cell Separation Microfluidic Device
}

\author{
https://doi.org/10.3991/ijoe.v13i06.7081 \\ Amjad Gawanmeh \\ Khalifa University, Abu Dhabi, UAE. \\ amjad.gawanmeh@kustar.ac.ae \\ Anas Alazzam \\ Khalifa University, Abu Dhabi, UAE. \\ anas.alazzamekustar.ac.ae \\ Bobby Mathew \\ UAE University, Al-Ain, UAE \\ bmathew@uaeu .ac.ae
}

\begin{abstract}
Blood cell separation microdevices are designed in biomedical engineering for the separation of particular cells from blood, such as cancer cells. The movement of blood microentities, especially abnormal ones, in a continuous flow microfluidic device is controlled by several forces. Therefore, understanding and guiding the movement of these microentities is a challenging problem. These cells are subject to different types of forces that result from natural or external effects. These forces include that due to gravity, virtual mass, buoyancy, dielectrophoresis, and inertia. Therefore, these are to be accounted for in any design or implementation of a system. In this paper we use formal analysis of a separation microdevice to model and verify the microenetit's movement and behavior at high level of abstraction while considering different types of forces. The dynamic behavior of the microentity can be modeled as a Markovian decision process to predict the trajectory of the same. This model can provide probabilistic analysis for the microentity movement in the microdevice under the effect of different types of forces.
\end{abstract}

Keywords-Cell separation, microentities, formal analysis, microdevice

\section{Introduction}

When microentities in a fluid medium are subjected to a non-uniform electric field, they respond to the generated force by moving within the medium. This behavior is known as Dielectrophoresis (DEP). The direction towards which microentities transverse depends on several factors, including the permittivity and conductivity of medium and microentity. In the design of separation devices for microentities, electrodes are integrated into the microchannel to subject microentities with DEP force normal to the direction of flow. As a result, microentities within the medium are pushed away/to from the electrodes $[1,2]$. 
There are several microparticles separation method proposed in the literature. Most of these are usually tested using a theoretical model as well as experimental data. Simulation, on the other hand, cannot provide full coverage for complex systems, since huge number of test cases are needed. Therefore, other complementary testing and verification techniques such as formal method are often used. Formal methods, particularly model checking, uses mathematical reasoning to model and verify different types of complex systems [3]. The objective is to make sure that design specifications are valid with regards to certain design requirements. Model checking [4] is an automatic formal verification technique that verifies whether a model of a system meets a given specification. Probabilistic model checking [5], on the other hand, is a variant of traditional model checking, where the probabilistic behavior of the given system is described using a Markovian model. This model can then be used to verify probabilistic properties. In this paper, we use PRISM probabilistic model checker which provides cutting-edge formal verification methods based on optimized techniques and has been used in several industrial contexts.

This paper presents a design and verification method for blood cell separation microdevice. The system is designed and implemented in the laboratory, and then formal performance analysis is conducted using probabilistic model checking. Probabilistic model checking will be used for the formal analysis of the movement of microentities in microdevices that are intended for cell separation. The proposed method can help improve the design by analyzing the effect of the position of the electrodes, and the deployed DEP force on the efficacy of the separation process. Experimental results show that certain position and forces can degrade the probability of successful separation of certain microentities.

\section{Related Work and Background}

Huang et al. [6] first proposed a model that can predict the trajectory of cells in microfluidic devices. The proposed method can determine the levitation height of cells subjected to external forces. Another work by Kralj et al. [7] modeled the trajectory of microparticles in a continuous flow microdevice, where separation is achieved based on the size of microparticles. Neculae et al. [8] did an analysis to predict microentity trajectory by considering all the forces associated with the particle. Lam et al. [9] developed a mathematical model to calculate the trajectory of microentities in a microfluidic device.

Model checking [4], sometimes called property checking, is a formal verification method that is commonly used to verify systems that can be modeled as statetransition graphs. It is commonly used to verify controllers, and communication protocols, where the verification is conducted exhaustively by exploring all the states of the model automatically. The properties to be verified are modeled in temporal logic. Model checking tools provide the failure trace in case a property is not valid. The main disadvantage of the model checking approach is that system states can grow exponentially and thus lead to the state-space explosion problem, which is the prob- 
lem of computationally handling the verification of system with a large state-space. A possible solution to this problem is to use a less complex model by using abstractions.

Probabilistic model checking [5] is a formal analysis method where the behavior of the system is described using a Markovian model and probabilistic properties can be defined and verified for this model. There are several probabilistic model checkers in the literature, such as PRISM [10]. We have used the PRISM model checker in this work as it supports a wide range of modeling options, such as Continuous Time Markov Chains (CTMCs), Discrete Time Markov Chains (DTMCs) and Markov Decision Processes (MDPs). The system to be verified in PRISM is modeled using the PRISM modeling and specification language. PRISM also supports a wide range of property specification languages, such as PCTL, CSL, LTL and PCTL. Once the given system is modeled with Continuous Time Markov Chain (CTMC) and is implemented in PRISM, the reliability properties are defined according to the needs and are verified to find the results. Another main reason for using the PRISM model checker in this work is to find failure and success probabilities about abnormal cells separation using microdevices.

The work in $[1,2]$ proposed a microfluidic device for the separation of abnormal cells from blood. The microdevice is designed by interdigitating electrodes on the bottom surface of the microchannel. The method was formally validated in [11] using model checking. The work in [12] presented validated dynamic model for predicting the path of microparticles subjected to a dielectrophoretic field, on a microfluidic device. In addition, the authors in [13] used switching of a focused stream of microsized particles in order to guide cells to desired locations inside a continuous flow microfluidic device. This paper extends pervious work by providing probabilistic analysis of the cell separation process in the microdevice. Despite the existence of several methods for testing and analysis of medical systems [14], to the best of our knowledge, this is the first attempt to apply probabilistic analysis on such system.

\section{Design of a Blood Cell Separation Microdevice}

Fig. 1 illustrates the different types of forces that are considered in the microchannel adopted in this work. The design is based on a repeating unit of the microchannel represented under steady state conditions, the electric field and electric potential inside this repeating unit are provided by Khoshmanesh et al. [15] and Zhang et al.[16]. The system specifications assume that there are no electric charges inside the microchannel. The force due to DEP depends on the gradient of the electric field and thus the need for non-uniform electric field. The force due to DEP also depends on the radius and electrical properties of the microentities, the electrical properties of the medium, and the applied frequency. The forces in Fig. 1 are defined as drag $\left(\boldsymbol{F}_{\text {Drag }}\right)$, DEP force $\left(\boldsymbol{F}_{\boldsymbol{D E P}}\right)$, and virtual mass force, $\left(\boldsymbol{F}_{\boldsymbol{v m}}\right)$ [1]. This model is based on similar assumptions that were initially provided in [17]. First, the microentities are assumed to be spherical, rigid, and its radius is very much smaller than the dimensions of the microchannel. The method assumes that there is no interactions between microentities. 


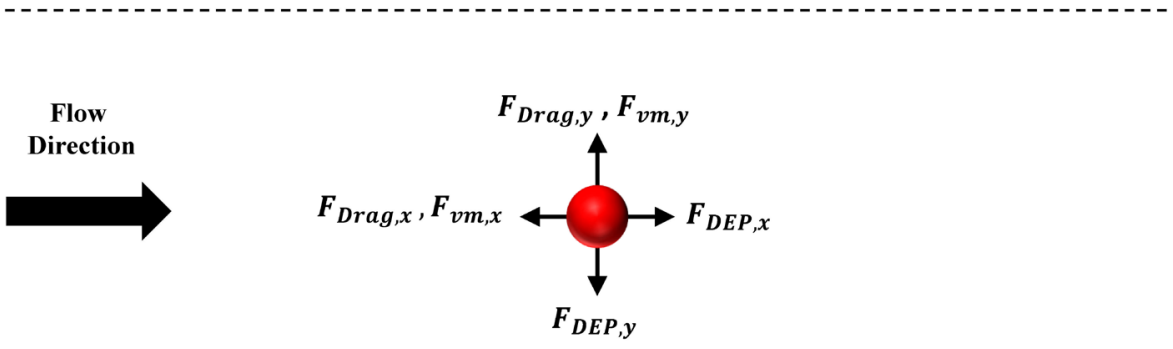

Fig. 1. Schematic of a section of the forces in blood cell separation microdevice [1].

According to Fig. 1, a microentity flows in the microchannel is affected by eight different forces. Some of these forces are imposed by the fluid flow, such as $\boldsymbol{F}_{\boldsymbol{v}}$. The main force that will be used to guide the microentities into the separation path is enforced through $\boldsymbol{F}_{\boldsymbol{D E P}}$. In our formal analysis, we assume that $\boldsymbol{F}_{\boldsymbol{D E P} \boldsymbol{P}}$ is controlled by the separation device, and the rest are controlled by other factors, such as field flow and location of the microentity. In the next section, we provide formal modeling and analysis for the separation system.

Fig. 2 below shows a view of separated abnormal cells from blood cells. We observed that some abnormal cells could not be captured in the separation process. Therefore, in the next step, we intend to conduct performance analysis on the design, which will help provide statistical information about the ability of the microdevice to capture abnormal cells. This can help in improving the performance of the microdevice by changing certain design parameters such as electric field strength, frequency or electrode position and dimensions.

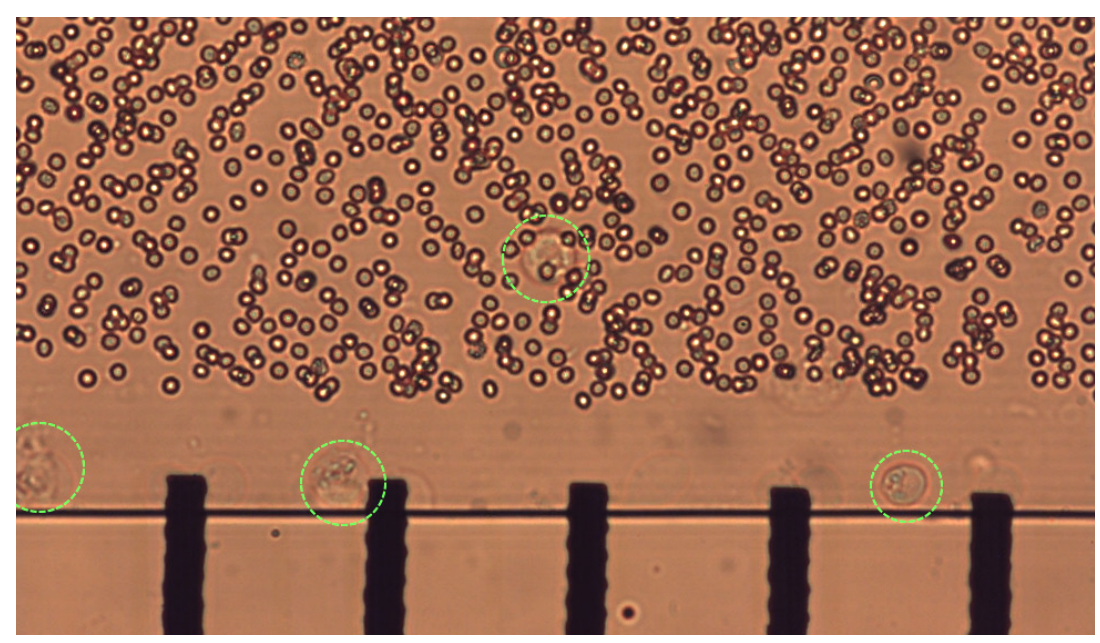

Fig. 2. Separation process of abnormal cells within the microfluidic flow. 


\section{Formal Probabilistic Analysis of Separation Microdevice}

Formal methods have proved to provide a complete coverage, and are becoming fundamental for the certification of different types of systems. Model checking [4] is a method that can be used for the modeling and design of state based systems. The system is first modeled, and then, this model verified exhaustively and automatically against certain specifications.

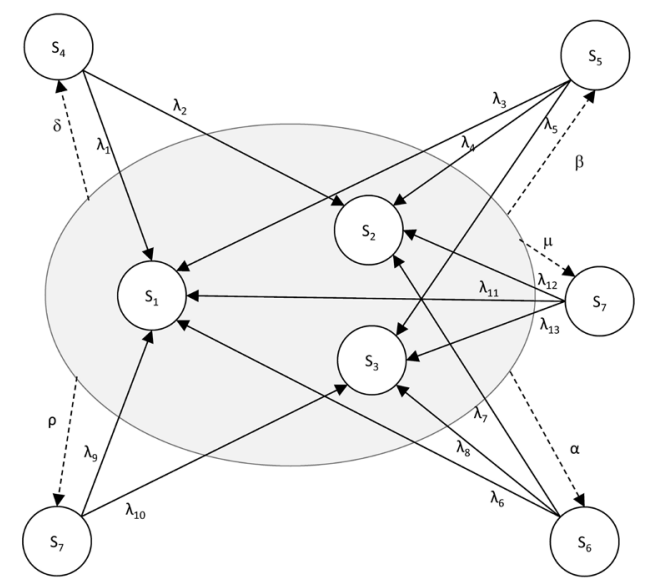

Fig. 3. The behavior of the separation device modeled with MDP.

PRISM model checker is used in this work to develop and verify a model for the microdevice. It supports probabilistic verification and has been used in several industrial contexts. Therefore, we will use it in order to model and verify the separation microdevice. First, a decision process is used to model the behavior of the microfluidic device, as illustrated in Fig. 3. Next, probabilistic analysis is conducted on the model for the state transaction probabilities as specified in Table 1. Fig. 4 shows the probability of successful separation vs. initial microentity position. In addition, Fig. 5 shows the probability of successful separation vs. initial microentity speed for different values of initial position. Results show that the system is affected by these parameters.

Table 1. State Transitional Probabilities for the MDP of the separation microdevice

\begin{tabular}{|c|c||c|c|}
\hline State Transitions & Probability & State Transitions & Probability \\
\hline$\lambda_{0}$ & 1 & $\lambda_{7}$ & 0.08 \\
\hline$\lambda_{1}$ & 0.02 & $\lambda_{8}$ & $1-\lambda_{6}-\lambda_{7}$ \\
\hline$\lambda_{2}$ & $1-\lambda_{1}$ & $\lambda_{9}$ & 0.05 \\
\hline$\lambda_{3}$ & 0.15 & $\lambda_{10}$ & $1-\lambda_{9}$ \\
\hline$\lambda_{4}$ & 0.05 & $\lambda_{11}$ & 0.60 \\
\hline$\lambda_{5}$ & $1-\lambda_{3}-\lambda_{4}$ & $\lambda_{12}$ & 0.20 \\
\hline$\lambda_{6}$ & 0.15 & $\lambda_{13}$ & $1-\lambda_{11}-\lambda_{12}$ \\
\hline
\end{tabular}




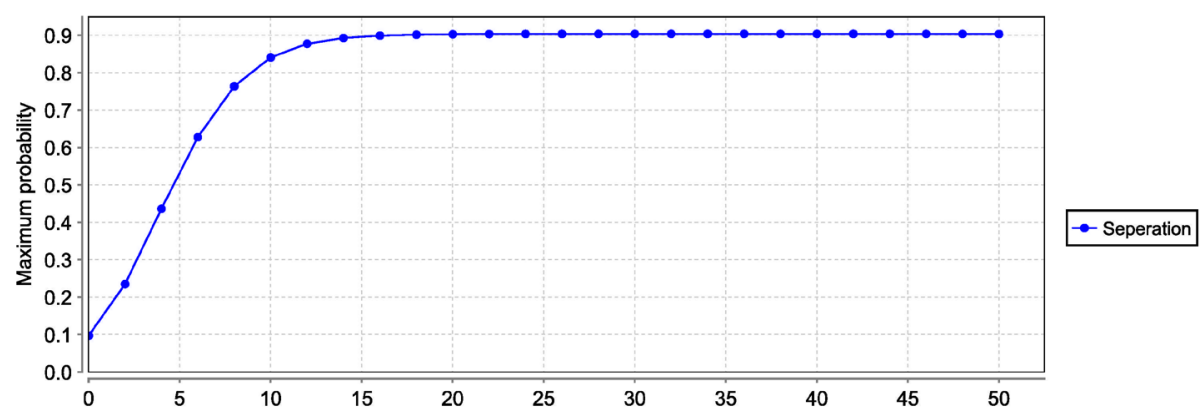

Fig. 4. Probability of successful separation vs. initial position of the microentity.

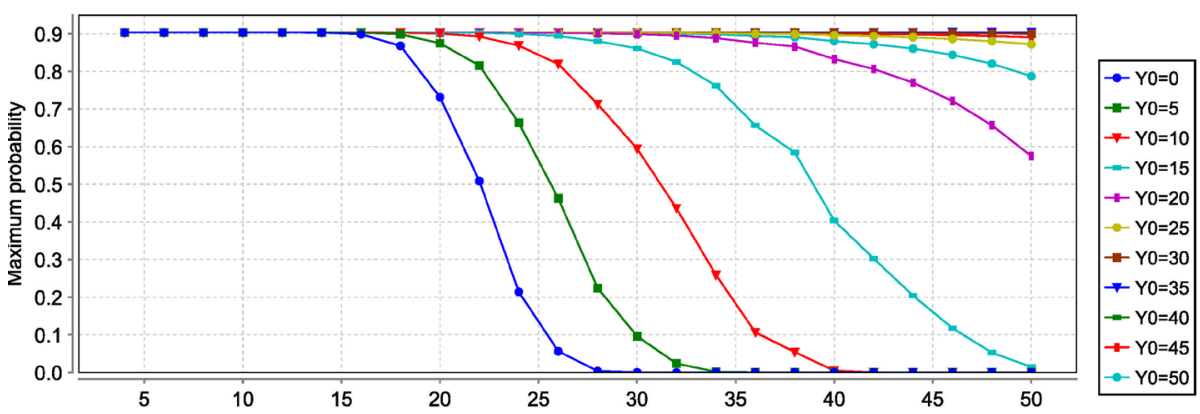

Fig. 5. Probability of successful separation vs. initial speed for different initial positions.

\section{Conclusion and Discussion}

Microentites' separation devices are designed and tested through mathematical models and experimental measurements. However, it will be very useful if such devices can be validated at early stage of the design process, where several parameters can be considered to enhance their efficiency. This paper presented a design and verification method for blood cell separation microdevice. First, the system is designed and implemented in the laboratory, and then formal performance analysis is conducted using probabilistic model checking. The main contributions of the paper include the development of the Markovian model microdevice based on the behavior of the microentity in the microdevice, the implementation of the microdevice, and the formal probabilistic analysis of the microdevice. The results show that the proposed method is scalable and practical. In addition, it can verify many novel reliability aspects compared to conventional papers and pencils analysis. Both experimental and formal analysis results show that the microdevice can successfully separate microentities with finite probabilities, based on the initial conditions of the particles, such as initial forces and position in the fluid. The proposed method in this paper can help identify and verify several important design parameters that help enhance the efficiency of similar microentity separation systems. To the best of our knowledge, this is 
the first novel work that combines formal probabilistic analysis and microdevices in the literature.

\section{Acknowledgment}

This work is financially supported by seed-grant funding from UAE University, Al Ain, Abu Dhabi (grant \# 31N264) and the seed funding program from Al Jalila Foundation, Dubai, UAE, (grant \# AJF201618).

\section{$7 \quad$ References}

[1] B. Mathew, A. Alazzam, M. Abutayeh, A. Gawanmeh, and S. Khashan, "Modeling the trajectory of microparticles subjected to dielectrophoresis in a microfluidic device for field flow fractionation," Chemical Engineering Science, vol. 138, pp. 266-280, 2015. https://doi.org/10.1016/j.ces.2015.07.014

[2] B. Mathew, A. Alazzam, S. Khashan, and M. Abutayeh, "Lab-on-chip for liquid biopsy (LoC-LB) based on dielectrophoresis," Talanta, vol. 164, pp. 608-611, 2017. https://doi.org/10.1016/j.talanta.2016.11.008

[3] P. Boca, J. P. Bowen, and J. I. Siddiqi, Formal methods: state of the art and new directions vol. 1: Springer, 2010. https://doi.org/10.1007/978-1-84882-736-3

[4] C. Baier, J.-P. Katoen, and K. G. Larsen, Principles of model checking: MIT press, 2008.

[5] M. Kwiatkowska, G. Norman, and D. Parker, "PRISM: probabilistic model checking for performance and reliability analysis," ACM SIGMETRICS Performance Evaluation Review, vol. 36, pp. 40-45, 2009. https://doi.org/10.1145/1530873.1530882

[6] Y. Huang, X.-B. Wang, F. F. Becker, and P. Gascoyne, "Introducing dielectrophoresis as a new force field for field-flow fractionation," Biophysical journal, vol. 73, pp. 1118-1129, 1997. https://doi.org/10.1016/S0006-3495(97)78144-X

[7] J. G. Kralj, M. T. Lis, M. A. Schmidt, and K. F. Jensen, "Continuous dielectrophoretic size-based particle sorting," Analytical chemistry, vol. 78, pp. 5019-5025, 2006. https://doi.org/10.1021/ac0601314

[8] A. Neculae, C. G. Biris, M. Bunoiu, and M. Lungu, "Numerical analysis of nanoparticle behavior in a microfluidic channel under dielectrophoresis," Journal of Nanoparticle Research, vol. 14, p. 1154, 2012. https://doi.org/10.1007/s11051-012-1154-4

[9] Y. C. Lam, S. H. Ling, W. Y. Chan, and K. S. Chian, "Dielectrophoretic cell motion model over periodic microelectrodes with unit-cell approach," Microfluidics and Nanofluidics, vol. 18, pp. 873-885, 2015. https://doi.org/10.1007/s10404-014-1478-8

[10] M. Kwiatkowska, G. Norman, and D. Parker, "PRISM 4.0: Verification of probabilistic real-time systems," in International Conference on Computer Aided Verification, 2011, pp. 585-591. https://doi.org/10.1007/978-3-642-22110-1 47

[11] A. Gawanmeh, A. Alazzam, and B. Mathew, "Formal Verification of a Microfluidic Device for Blood Cell Separation," Scalable Computing: Practice and Experience, vol. 17, pp. 227-236, 2016.

[12] B. Mathew, A. Alazzam, S. Khashan, and B. El-Khasawneh, "Path of microparticles in a microfluidic device employing dielectrophoresis for hyperlayer field-flow fractionation," Microsystem Technologies, vol. 22, pp. 1721-1732, 2016. https://doi.org/10.1007/s00542$\underline{016-2811-9}$ 
[13] B. Mathew, A. Alazzam, G. Destgeer, and H. J. Sung, "Dielectrophoresis based cell switching in continuous flow microfluidic devices," Journal of Electrostatics, vol. 84, pp. 63-72, 2016. https://doi.org/10.1016/j.elstat.2016.09.003

[14] A. Gawanmeh, H. Al-Hamadi, M. Al-Qutayri, S.-K. Chin, and K. Saleem, "Reliability analysis of healthcare information systems: State of the art and future directions," in $E$ health Networking, Application \& Services (HealthCom), 2015 17th International Conference on, 2015, pp. 68-74.

[15] K. Khoshmanesh, S. Nahavandi, S. Baratchi, A. Mitchell, and K. Kalantar-zadeh, "Dielectrophoretic platforms for bio-microfluidic systems," Biosensors and Bioelectronics, vol. 26, pp. 1800-1814, 2011. https://doi.org/10.1016/j.bios.2010.09.022

[16] C. Zhang, K. Khoshmanesh, A. Mitchell, and K. Kalantar-Zadeh, "Dielectrophoresis for manipulation of micro/nano particles in microfluidic systems," Analytical and bioanalytical chemistry, vol. 396, pp. 401-420, 2010. https://doi.org/10.1007/s00216-009-2922-6

[17] E. Loth, "Numerical approaches for motion of dispersed particles, droplets and bubbles," Progress in Energy and Combustion Science, vol. 26, pp. 161-223, 2000. https://doi.org/10.1016/S0360-1285(99)00013-1

\section{Authors}

Amjad Gawanmeh is member of IEEE. He is an assistant professor at Khalifa University, Department of Electrical and Computer Engineering, Abu Dhabi, UAE.

Anas Alazzam is an assistant professor at Khalifa University, Department of Electrical and Computer Engineering, Abu Dhabi, UAE.

Bobby Mathew is an assistant professor at UAE University, Department of Electrical and Computer Engineering, P O Box 15551, Al-Ain, UAE.

Article submitted 28 April 2017. Published as resubmitted by the authors 03 June 2017. 